\title{
SYMMETRIZABLE AND RELATED SPACES
}

\author{
BY
}

PETER W. HARLEY III AND R. M. STEPHENSON, JR.(1)

\begin{abstract}
A study is made of a family of spaces which contains the symmetrizable spaces as well as many of the well-known examples of perfectly normal spaces.
\end{abstract}

1. Introduction. For some time mathematicians have been interested in the questions: Is every perfect compact Hausdorff space hereditarily separable? (As in [HM], a space will be called perfect if each of its closed sets is a $G_{\delta}$.) If each discrete subspace of a compact Hausdorff space is countable, is the space hereditarily Lindelöf? Is every regular, perfect, countably compact space compact? (For some recent results concerning these questions, see $[0],[T],[W]$, and [JKR].)

In this paper we define and study a family $F$ of spaces for which each of these questions has an affirmative answer. It will be seen that $F$ is quite large and that, consequently, the results obtained will apply to all semimetrizable spaces and all symmetrizable spaces (defined below) and to a number of familiar examples, e.g., the Michael line, the Sorgenfrey line, and the top and bottom of the lexicographically ordered square.

In $\$ 2 F$ is defined, and some properties of spaces in $F$ are derived, including a simple example of a symmetrizable space in which no point is a $G_{\delta}$. In $\S 3$ proofs are given that every countably compact space in $F$ is compact, and that a space $X$ in $F$ is hereditarily Lindelöf if and only if each discrete subspace of $X$ is countable. These two theorems strengthen analogous results of S. Nedev [N1] obtained for symmetrizable spaces. We also prove that a first countable Hausdorff space in $F$ is hereditarily separable if and only if it is hereditarily Lindelöf, and A. V. Arhangel'skii's theorem [A2, p. 126] that a compact Hausdorff symmetrizable space is first countable (and hence metrizable) is extended to: Every compact Hausdorff $F$-space is first countable. $\$ 4$ contains a family $L$ of hereditarily separable compact Hausdorff $F$-spaces such that $|L|>\exp \left(\kappa_{0}\right)$ and no two spaces in $L$ are homeomorphic. Using $L$, one can obtain an $F$-analogue of the theorem of

Presented to the Society, January 16, 1974; received by the editors December 27, 1973 and, in revised form, December 4, 1974.

AMS (MOS) subject classifications (1970). Primary 54D55, 54E25; Secondary 54A25, 54B10, 54D15, 54D20.

(1) Both authors' research was supported by grants from the University of South Carolina. 
V. Filippov [F1] that there exists no universal perfect compact Hausdorff space. $\$ 5$ contains an example of a separable compact Hausdorff F-space in which there is a discrete subspace of cardinality $\exp \left(\aleph_{0}\right)$. In the final section, some suitable restrictions are considered under which symmetrizability is countably productive, and under which necessary and sufficient conditions can be given that a product space be an F-space. We conclude the paper by proving that for a symmetrizable space $X$ in which each point is a regular $G_{\delta}, X$ is locally compact if and only if for every symmetrizable Hausdorff space $Y, X \times Y$ is symmetrizable.

2. Some examples and properties of $F$-spaces. For a topological space $X$, we will write $X \in F$ and say that $X$ is an F-space if there exists a mapping $B: \mathrm{N} \times$ $X \rightarrow P(X)$, where $P(X)$ denotes the power set of $X$, satisfying the following conditions.

(i) Each $B(n+1, x) \subset B(n, x)$ and $\{x\}=\bigcap\{B(i, x) \mid i \in \mathrm{N}\}$.

(ii) A set $V \subset X$ is open if and only if for every $x \in V$, there exists $n \in N$ such that $B(n, x) \subset V$.

(iii) For each closed set $F$ and point $x \in X \backslash F$, there exists $i \in \mathbf{N}$ such that for every point $y \in B(i, x) \backslash\{x\}$, there exists $k(y) \in N$ for which $\{x, y\}$ is not contained in $\bigcup\{B(k(y), f) \mid f \in F\}$.

Any mapping $B: \mathrm{N} \times X \rightarrow P(X)$ such that (i), (ii), and (iii) hold will be called an F-system for the space $X$, and given a set $F \subset X$, we will write $B(k, F)$ for $\bigcup\{B(k, f) \mid f \in F\}$. An $F$-system $B$ for $X$ will be called a neighborhood $F$ system for $X$ if each $B(n, x)$ is a neighborhood of $x$. A space that admits a neighborhood F-system is said to be a neighborhood F-space.

Conditions (i) and (ii) just require that every F-space satisfy the weak first axiom of countability of Arhangel'skii [A2, p. 129] and be a $T_{1}$-space. Note that every mapping $B: \mathrm{N} \times X \rightarrow P(X)$ satisfying (i) determines a unique topology on $X$ such that (ii) is satisfied. Condition (iii) is a weakening of the property of any metric space that each point $x$ has a neighborhood base $\{B(n, x) \mid n \in N\}$ satisfying $x \in B(n, y)$ if and only if $y \in B(n, x)$. Let us now give some examples of spaces belonging to $F$.

In Examples 2.1 and 2.2, conditions (i), (ii), and (iii) are easily satisfied for obvious choices of $B(n, x)$.

Examples 2.1. Let $M$ be the Michael line, the set $\mathbf{R}$, topologized as follows: each point of $R \backslash Q$ is isolated; a basic open neighborhood of a point $q \in Q$ is any open interval containing $q$.

EXAMPLE 2.2. Let $S$ be the Sorgenfrey line, that is, $\mathbf{R}$ with the topology induced on $\mathbf{R}$ by all sets of the form $[x, y)$.

EXAMPLE 2.3. Let $L=[0,1] \times\{0,1\}$, and call a subset $V$ of $L$ open if and only if $V$ is a union of sets of the form 


$$
L \cap(((x, y] \times\{0\}) \cup([x, y) \times\{1\})),
$$

i.e., let $L$ have the topology it inherits from the lexicographically ordered square. For $n \in N$ and $x \in[0,1]$, define

$$
\begin{aligned}
& B(n,(x, 0))=\{(x, 0)\} \cup(L \cap((x-1 / n, x) \times\{0,1\})), \\
& B(n,(x, 1))=\{(x, 1)\} \cup(L \cap((x, x+1 / n) \times\{0,1\})) .
\end{aligned}
$$

Clearly, conditions (i) and (ii) are satisfied, and for an isolated point, (iii) holds vacuously. For a nonisolated point $p=(x, y)$, if one takes $F=L \backslash B(n, p)$ and $i$ $\in \mathrm{N}$ with $i>n$, then the conclusion of (iii) is satisfied for any point $\left(x^{\prime}, y^{\prime}\right) \in$ $B(i, p) \backslash\{p\}$ and $k \in N$ for which $1 / k \leqslant \min \left\{1 / n-1 / i,\left|x-x^{\prime}\right|\right\}$.

EXAMPLE 2.4. Let $E$ be the double interval of Alexsandrov, the set $[0,1]$ $\times\{0,1\}$, topologized as follows: each subset of $[0,1] \times\{0\}$ is open; if $x \in$ $[0,1]$, then a neighborhood of the point $(x, 1)$ is any set $V(U)=((U \backslash x\}) \times\{0\})$ $U(U \times\{1\})$, where $U$ is a neighborhood of $x$ in the usual topology on $[0,1]$. For $n \in \mathrm{N}$ and $x \in[0,1]$, let $B(n,(x, 0))=\{(x, 0)\}$ and $B(n,(x, 1))=V(U)$ for $U=\{t \in[0,1]|| x-t \mid<1 / n\}$.

Each of the examples above is a neighborhood F-space. The next one is not. Before we describe it, a definition is needed.

A symmetric for a topological space $X$ is a mapping $d: X \times X \rightarrow[0, \infty)$ such that

(1) For all $x, y \in X, d(x, y)=d(y, x)$, and $d(x, y)=0$ if and only if $x=y$.

(2) A set $V \subset X$ is open if and only if for each $x \in V$ there exists $n \in \mathbf{N}$ such that $V$ contains the set $B(n, x)=\{y \in X \mid d(x, y)<1 / n\}$.

A space $X$ which admits a symmetric is said to be symmetrizable, and if, in addition, each $B(n, x)$ is a neighborhood of $x$, then $X$ is said to be semimetrizable and $d$ is called a semimetric for $X$. Equivalently, $X$ is semimetrizable via $d$ provided that for $x \in X, A \subset X$, and $d(x, A)=\inf \{d(x, a) \mid a \in A\}$, the condition $x$ $\in \bar{A}$ if and only if $d(x, A)=0$ is satisfied.

It is easy to check that every symmetrizable space is an $F$-space, and that, conversely, every space $X$ with an $F$-system $B$ satisfying, for all $x, y \in X$ and $n \in \mathrm{N}$,

(iii)' $x \in B(n, y)$ if and only if $y \in B(n, x)$, is symmetrizable. (To verify the latter assertion, put $d(x, x)=0$, and for $y \neq x, d(x, y)=1 / \min \{n \mid y \notin B(n, x)\}$.)

Later in this section short proofs will be given that none of Examples 2.12.4 is symmetrizable (this fact is probably fairly well known-e.g., see R. W. Heath [H, p. 154]). The next example is a symmetrizable Hausdorff space which is not semimetrizable (a similar example appears in [A2, Example 2.2]).

EXAMPLE 2.5. Let $H=\mathbf{R}$, topologized as follows: For $x \in \mathbf{R} \backslash\{0\}$, a neighborhood of $x$ is any open interval containing $x$. A neighborhood of 0 is any set 
of the form

$$
\{0\} \cup \bigcup\{(z-a(z), z+a(z))|z \in \mathbf{Z},| z \mid \geqslant n, \text { and } a(z)>0\},
$$

where $n \in \mathrm{N}$. Let $d: H \times H \rightarrow[0, \infty)$ be such that $d(x, y)=|x-y|$ for $x y \neq 0$; $d(z, 0)=d(0, z)=1 /|z|$ for $z \in Z \backslash\{0\} ; d(x, 0)=d(0, x)=1$ if $x \notin Z$; and $d(0,0)=0$.

The space $H$ is symmetrizable via the symmetric $d$. Since 0 does not have a countable neighborhood base, $H$ cannot be a neighborhood $F$-space.

Let us recall that a topological space $X$ is said to be Fréchet provided that for every $A \subset X$ and point $x \in \bar{A}$ there exists a sequence $\left\{a_{n}\right\}$ in $A$ such that $a_{n} \rightarrow x$.

If $X$ is a space with $F$-system $B$, we will say that a sequence $\left\{x_{n}\right\}$ in $X B$ converges to a point $x \in X$ if for each $m \in \mathbf{N}$ there exists $k \in \mathbf{N}$ such that for all $n \in \mathrm{N}$ with $n \geqslant k, x_{n} \in B(m, x)$. The mapping $B$ will be said to satisfy (*) provided that for every sequence $\left\{x_{n}\right\}$ and point $x$ in $X$, if $\left\{x_{n}\right\}$ converges to $x$, then $\left\{x_{n}\right\}$ also $B$-converges to $x$. (Clearly, if $\left\{x_{n}\right\} B$-converges to $x$ then always $\left\{x_{n}\right\}$ converges to $x$.)

THEOREM 2.6. Let $X$ be an F-space via $B$. Then the following hold.

(a) $B$ is a neighborhood F-system for $X$ if and only if $X$ is Fréchet and $B$ satisfies (*).

(b) If every compact subspace of $X$ is closed, then $B$ satisfies (*).

(c) If $X$ is Hausdorff, then $B$ is a neighborhood F-system for $X$ if and only if $X$ is Fréchet.

Proof. The proof of (a) is straightforward, and (c) is an immediate consequence of (b).

To establish (b), suppose there exist a sequence $\left\{x_{n}\right\}$ in $X$ and a point $x \in$ $X$ such that $x_{n} \rightarrow x$ but $\left\{x_{n}\right\}$ does not $B$-converge to $x$. Passing to a subsequence if necessary, we may assume that there is an $m \in \mathrm{N}$ with $C \cap B(m, x)=\varnothing$, where $C$ is the set of values of $\left\{x_{n}\right\}$. If the compact set $K=C \cup\{x\}$ is closed, then for each point $y \in X \backslash K$ there exists $k(y)$ with $C \cap B(k(y), y)=\varnothing$, but then it would follow from condition (ii) and the equation $X \backslash C=B(m, x) \cup$ $\bigcup\{B(k(y), y) \mid y \in X \backslash K\}$ that $C$ is closed, whereas $x \notin C$ and $x_{n} \rightarrow x$.

We observe that if $d$ is a symmetric for $X$ and $B(n, x)=\{y \in X \mid d(x, y)<$ $1 / n\}$, then (*) is equivalent to the following: $x_{n} \rightarrow x$ implies $d\left(x_{n}, x\right) \rightarrow 0$.

REMARK 2.7. Since the space $H$ in Example 2.5 is symmetrizable and Hausdorff but not semimetrizable, it follows from Theorem 2.6 that $H$ is not a Fréchet space. In particular, one can show that 0 is in the closure of $H \backslash \mathrm{Z}$, but that no sequence in $H \backslash \mathbf{Z}$ converges to 0 . For if there were such a sequence $\left\{x_{n}\right\}$, then we would have $d\left(x_{n}, 0\right) \rightarrow 0$ by Theorem 2.6 , whereas $d(0, H \backslash Z)=1$. 
A subset $A$ of a space $X$ is said to be sequentially closed if for every sequence $\left\{a_{n}\right\}$ in $A$ and point $x \in X$, if $a_{n} \rightarrow x$ then $x \in A$. If every sequentially closed subset of $X$ is a closed subset of $X$, then $X$ itself is a sequential space.

While not every F-space is Fréchet, at least the following is true.

THEOREM 2.8. Every F-space is a sequential space.

In fact, every weakly first countable space is sequential.

Notation. Given an $F$-system $B$ for a space $X$, a subset $Y$ of $X$, and a point $y \in Y, B_{Y}(n, y)$ will denote $Y \cap B(n, y)$.

THEOREM 2.9. Let $X$ be a space having an F-system $B$, and let $Y$ be a subspace of $X$. Then $B_{Y}$ is an F-system for $Y$ if any of the following conditions is satisfied.

(a) $B$ is a neighborhood F-system for $X$.

(b) $Y$ is open or closed in $X$.

(c) $Y$ is a sequential space and $B$ satisfies (*).

Proof. Clearly, for any subspace $Y, B_{Y}$ satisfies (i) and (iii).

To see that $B_{Y}$ satisfies (ii), consider any open subset $V$ of $Y$. Choose an open subset $W$ of $X$ such that $W \cap Y=V$, and for each $x \in W$ choose $n(x) \in \mathbf{N}$ with $B(n(x), x) \subset W$. Then for every point $v \in V, V \supset B_{Y}(n(v), v)$.

Conversely, suppose that $F$ is a subset of $Y$ such that for every point $y \in$ $Y \backslash F$ there exists $m(y)$ with $B_{Y}(m(y), y) \cap F=\varnothing$. We wish to show that if (a), (b), or (c) holds, then $F$ is a closed subset of $Y$.

If (a) holds, then $Y \backslash F$ is obviously an open subset of $Y$.

(b) If $Y$ is open, choose, for each $y \in Y \backslash F$, an integer $p(y) \geqslant m(y)$ for which $B(p(y), y) \subset Y$. Then $Y \backslash F=\bigcup\{B(p(y), y) \mid y \in Y \backslash F\}$, so $Y \backslash F$ is an open subset of $X$. If $Y$ is closed, choose, for each $x \in X \backslash Y$, an integer $m(x)$ for which $B(m(x), x) \cap Y=\varnothing$. Then $X \backslash F=\bigcup\{B(m(x), x) \mid x \in X \backslash F\}$ and $X \backslash F$ is an open subset of $X$.

(c) It follows from (*) and the existence of the numbers $m(y), y \in Y \backslash F$, that $F$ is a sequentially closed subset of $Y$. Hence $F$ is a closed subset of $Y$.

Thus condition (ii) holds, and $B_{Y}$ is an $F$-system for $Y$.

While membership in $F$ is hereditary for first countable Hausdorff spaces, an analogous result does not hold for all Hausdorff spaces.

ExAmple 2.10. Let $H$ be as in Example 2.5. Take $A=H \backslash Z$ and $Y=\{0\}$ $\cup A$. Then $A$ is a sequentially closed but not closed subset of $Y$, so by Theorem 2.8, $Y \notin F$.

REMARK 2.11. Let $W=\{0\} \cup N \cup\{m+1 / n \mid m, n \in \mathrm{N}, n \geqslant 2\}$, with the topology it inherits as a closed subspace of $H$. In the study of symmetrizable 
spaces which fail to be semimetrizable, the spaces $H$ and $W$ are quite useful, have many properties in common, and arise naturally-see Lemmas 6.10-6.11 and [FH]. Several other authors have independently noticed the usefulness of a space, called $M$ in [F3], which is homeomorphic with $W$-see [A1] and [F3]; specifically, our observation that $W$ is a sequential space and that no sequence in $W \backslash \mathbf{N}$ converges to 0 was also made in [F3, Example 5.1].

The next example shows that (*) cannot be eliminated from (c) of Theorem 2.9, even for a first countable space.

ExAmple 2.12. Let $D=\mathrm{N}$, with the cofinite topology. Define $d: D \times D$ $\rightarrow[0, \infty)$ by: for each $n, d(n, n)=0$; for $m \neq n, d(m, n)=1$ if both $m$ and $n$ are even integers, and, otherwise, $d(m, n)=1 /|m-n|$.

It is easy to see that $D$ is symmetrizable via $d$; however, if $Y$ is the set of even integers in $D$, then $d$ induces the discrete topology on $Y$. Hence, by Theorem 2.9, since $D$ is first countable, $d$ does not satisfy (*) and $d$ is not a semimetric for $D$. In particular, $2 n \rightarrow 2$ but $d(2 n, 2) \nrightarrow 0$.

If one defines $d^{\prime}: D \times D \rightarrow[0, \infty)$ by the rule $d^{\prime}(m, n)=1 /|m-n|$ if $m \neq n$, and $d^{\prime}(m, m)=0$, then $d^{\prime}$ is a semimetric for $D$.

It is natural to ask if there is a symmetrizable space $C$ such that no $F$. system for $C$ satisfies (*). According to Theorem 2.6(a) one could establish the existence of such a space by finding a symmetrizable Fréchet space having no neighborhood $F$-system. We now give a simple example of such a space $C$ which also has the property that no point of $C$ is a $G_{\delta}$. As far as the authors know, this is the first known example of a symmetrizable space with the latter property -an example of a symmetrizable space containing one point that is not a $G_{\delta}$ was given by D. Bonnett in [B2]. (While another example in [B2] showed that not every Hausdorff symmetrizable space is perfect, it is apparently still not known if there is a Hausdorff symmetrizable space containing a point that is not a $G_{\delta}$.)

EXAMPLE 2.13. Let $C$ be the space consisting of the countable ordinals, with the cofinite topology. Then $C$ is a symmetrizable Fréchet space in which no point is a $G_{\delta}$.

Proof. Obviously $C$ is Fréchet and has no $G_{\delta}$ points.

A symmetric for $C$ will be constructed by transfinite induction. Let $b \in C$ and suppose that we have already defined a one-to-one mapping $f_{a}$ of $[0, a]$ into $\mathbf{N}$ for each $a \in C$ with $a<b$. To define $f_{b}$, if $b=c+1$ for some $c$, then let $f_{b}:[0, b] \rightarrow \mathrm{N}$ be determined by the rule $f_{b}(b)=1$ and $f_{b}(a)=f_{c}(a)+1$ for each $a<b$. In case $b$ has no immediate predecessor, just take $f_{b}$ to be a one-to-one mapping of $[0, b]$ into $N$. Next define $d: C \times C \rightarrow[0, \infty)$ by

$$
d(a, b)=d(b, a)=\left\{\begin{array}{l}
0 \text { if } a=b \\
1 / f_{b}(a) \text { if } a<b .
\end{array}\right.
$$


In order to prove that the space $C$ is symmetrizable via $d$, it suffices to show that if $F$ is a subset of $C$ with $d(c, F)>0$ for every $c \in C \backslash F$, then either $F$ is finite or $F=C$.

Case 1. There exists $t \in C$ with $F \supset\{x \in C \mid x \geqslant t\}$. Then, clearly, for any $c<t, d(c,\{t+i \mid i \in \mathrm{N}\})=0$, so $c \in F$ and $F=C$.

Case 2. Suppose that no such $t$ exists. Then either $F$ is finite or there exists $c \in C \backslash F$ such that $F \cap[0, c)$ is infinite. But for such a point $c$, $f_{c}(F \cap[0, c))$ would be infinite and one would have $d(c, F)=0$. Thus $F$ must be finite.

Let us now note why none of the spaces in Examples 2.1-2.4 is symmetrizable. Since each is first countable and Hausdorff, it suffices, e.g., by Theorem 2.9, to show that each space fails to be semimetrizable. That these familiar examples are not semimetrizable is probably known to many; in any case, for completeness and also for later use, we include the following short proofs.

It is well known that Examples 2.3 and 2.4 are compact but not second countable (although the space $L$ is perfect and hereditarily separable). Since by a result of Niemytzki [N2] (see also Theorem 3.13) every compact Hausdorff semimetrizable space is metrizable, neither $L$ nor $E$ admits a semimetric.

In the Michael line $M$ the closed set $\mathbf{Q}$ cannot be a $G_{\delta}$, for if $\mathbf{Q}$ were, then it would also be a $G_{\delta}$ with respect to the usual topology on $\mathbf{R}$. On the other hand, it is known (and easy to check) that if a neighborhood F-system $B$ satisfies condition (iii)', then for each closed set $F$,

$$
F=\bigcap\{\text { interior of } B(n, F) \mid n \in \mathrm{N}\} \text {. }
$$

For the Sorgenfrey line $S$, suppose there exists a neighborhood $F$-system $B$ satisfying (iii)'. Then for each $s \in S$ there exists $k(s) \in \mathbf{N}$ such that $B(k(s), s)$ and the closed set $(-\infty, s)$ are disjoint. Choose $k \in \mathrm{N}$ such that the set $Y=$ $\{s \mid k(s)=k\}$ is uncountable. Then by (iii)' each $B(k, y), y \in Y$, contains exactly one point of $Y$, which is impossible since $S$ is hereditarily Lindelöf.

3. Equivalence of separability and compactness conditions in F-spaces. Examples show that spaces in $F$ can be quite different from metrizable spaces. Example 2.3 is perfect and compact Hausdorff but not second countable (for some other spaces of this type, see [V]); the space in Example 2.4 is compact Hausdorff but has uncountably many isolated points; and Example 3.12 is a noncompact, perfect, zero-dimensional Moore space in which every locally finite system of open sets is finite. We will prove below, however, that, as is the case with metrizable spaces, quite a number of generalizations of compactness are equivalent concepts for $F$-spaces.

A space is called $\aleph_{1}$-compact if each uncountable subset has a limit point. A subset $Y$ of a space $X$ will be called a discrete subspace of $X$ if $Y$, with the subspace topology it inherits from $X$, is a discrete space. If $B$ is an F-system for 
$X$, then a subset $Y$ of $X$ is said to be $B-k$ discrete, where $k \in \mathrm{N}$, provided that whenever $y, z \in Y$ with $y \neq z$, then $y \notin B(k, z)$ and $z \notin B(k, y)$.

LEMMA 3.1. Let $X$ be an $\aleph_{1}$-compact space with F-system $B$. Then every closed $B-k$ discrete subset $Y$ of $X$ is countable.

Proof. Suppose that $Y$ is uncountable. Since $X$ is $\aleph_{1}$-compact, $Y$ has a limit point $p$, and since $Y$ is closed, $p$ must be in $Y$.

Now $Y \backslash\{p\}$ is not closed, and the subspace $Y$ admits $B_{Y}$ as an $F$-system (by Theorem 2.9), so it follows from condition (ii) of the definition of an F-system that each $B_{Y}(n, p) \cap(Y \backslash\{p\}) \neq \varnothing$. In particular, $B(k, p) \cap(Y \backslash\{p\}) \neq \varnothing$, which contradicts the assumption that $Y$ is $B-k$ discrete.

LEMMA 3.2. Let $X$ be an $\aleph_{1}$-compact space with F-system $B$. Suppose that $(C,<)$ is a well-ordered subset of $X$ for which there exist open subsets $V(x)$ of $X$, $x \in C$, such that for each $x \in C$ and $n \in \mathrm{N}$ :

(1) $x \in V(x)$;

(2) if $t \in C \cap V(x)$ then $t \leqslant x$; and

(3) there exists $y \in B(n, x)$ with $y \neq x$ and with $y \notin \bigcup\{V(t) \mid t<x\}$.

Then $C$ is countable.

Proof. Let us suppose that there exists an uncountable subset $C$ of $X$ for which the statements above hold.

For each $x \in C$ choose $n(x) \in \mathrm{N}$ such that $B(n(x), x) \subset V(x)$, and select an $i(x) \geqslant n(x)$ provided by condition (iii) of the definition of an F-system, where (iii) is applied to the point $x$ and the closed set $X \backslash V(x)$. Choose an $i \in \mathrm{N}$ so that the set $Y(1)=\{x \in C \mid i(x)=i\}$ is uncountable.

For each $x \in Y(1)$, it follows from (3) above that there exists $y(x) \in$ $B(i, x) \backslash\{x\}$ with $y(x) \notin \bigcup\{V(t) \mid t<x\}$. Then by (iii), for each $x \in Y(1)$, there exist $k(y(x)) \in N$ and $z(x) \in\{x, y(x)\}$ such that $z(x) \notin B(k(y(x)), X \backslash V(x))$. Choose $k \in \mathbf{N}$ so that $Y(2)=\{z(x) \mid k(y(x))=k\}$ is uncountable.

Now each $z(x) \in Y(2)$ is in the open set $V(x)$ (since $y(x) \in B(i, x) \subset$ $B(n(x), x) \subset V(x))$, so there exists $j(x) \geqslant k$ with $B(j(x), z(x)) \subset V(x)$. Select $j \in$ $\mathrm{N}$ with $Y=\{z(x) \in Y(2) \mid j(x)=j\}$ uncountable.

Let us now observe that the set $Y$ is $B-j$ discrete. For consider any $z(x)$ and $z\left(x^{\prime}\right)$ in $Y$ with $x<x^{\prime}$. Because $B(j, z(x)) \subset V(x)$ and $z\left(x^{\prime}\right) \notin V(t)$ for any $t<x^{\prime}$, it follows that $z\left(x^{\prime}\right) \notin B(j, z(x))$. Similarly, since $z(x) \notin B(k, X \backslash V(x))$ and $z\left(x^{\prime}\right) \in$ $X \backslash V(x)$, and since $j \geqslant k$, we have $z(x) \notin B\left(j, z\left(x^{\prime}\right)\right)$.

By Lemma 3.1, $Y$ cannot be closed. Thus there is a point $b \in X \backslash Y$ such that every $B(m, b) \cap Y \neq \varnothing$. It follows from this and from the choice of $k$ that $b \in \bigcup\{V(x) \mid z(x) \in Y\}$, for, otherwise, one would have $B(k, b) \cap Y=\varnothing$.

Let $e$ be the first element of $C$ for which $z(e) \in Y$ and $b \in V(e)$. Choose 
any $p \in \mathbf{N}$ such that $p \geqslant k, B(p, b) \subset V(e)$, and $z(e) \notin B(p, b)$. Then $B(p, b) \cap$ $Y=\varnothing$, which is a contradiction.

For symmetrizable spaces, the next theorem is known [N1].

Theorem 3.3. Let $X$ be an F-space. The following are equivalent.

(a) $X$ is $\aleph_{1}$-compact.

(b) $X$ is a Lindelöf space.

Proof. (b) implies (a) is well known to be true for arbitrary spaces.

Suppose that $X$ is $\aleph_{1}$-compact but not Lindelöf, and let $V$ be an open cover of $X$ which has no countable subcover. Let $B$ be an $F$-system for $X$. Then there exist points $x(a)$ and sets $V(a) \in V$ such that, for each countable ordinal $a$ :

(1) $x(a) \in V(a)$;

(2) if $a<b$ then $x(b) \notin V(a)$; and

(3) for each $n \in \mathrm{N}$ there exists $y \in B(n, x(a))$ with $y \neq x(a)$ and with $y \notin$ $\bigcup\{V(b) \mid b<a\}$.

For suppose that $c$ is a countable ordinal, and we have chosen points $x(a)$ and sets $V(a) \in V$ satisfying (1)-(3) for all $a<c$. We wish to define $x(c)$ and $V(c)$ so that (1)-(3) hold for all ordinals $a \leqslant c$.

Let $V=\bigcup\{V(a) \mid a<c\}$. Since $V$ is a union of a countable subcollection of $V, X \backslash V$ must be uncountable and hence have a limit point $p$. Because $B$ induces the subspace topology on the closed set $X \backslash V$, it follows that each $B(n, p) \cap$ $(X \backslash V) \backslash\{p\} \neq \varnothing$. Let $x(c)=p$ and choose a set $V(c) \in V$ with $x(c) \in V(c)$. Clearly, (1), (2), and (3) hold for all $a \leqslant c$.

Thus the set $\{x(a)\}$ is uncountable. Appealing to Lemma 3.2, we obtain a contradiction.

Lfmma 3.4. Let $X$ be a $T_{1}$-space. The following are equivalent.

(a) Each discrete subspace of $X$ is countable.

(b) Each open subspace of $X$ is $\aleph_{1}$-compact.

THEOREM 3.5. Let $X$ be an F-space. The following are equivalent.

(a) Each discrete subspace of $X$ is countable.

(b) $X$ is hereditarily Lindelöf.

Proof. By Theorem 2.9, every open subspace of an F-space is an $F$-space, so by Theorem 3.3 and Lemma 3.4, it follows from (a) that every open subspace of $X$ is Lindelöf.

Corollary 3.6. Let $X$ be a perfect F-space. The following are equivalent.

(a) Each discrete subspace of $X$ is countable.

(b) $X$ is a Lindelöf space.

(c) $X$ is $\aleph_{1}$-compact.

In [AU] Alexsandrov and Urysohn proved that every perfect compact Haus- 
dorff space has no more than $\exp \left(\aleph_{0}\right)$ closed sets. For neighborhood $F$-spaces, a similar result is true.

Corollary 3.7. Let $X$ be a perfect neighborhood F-space.

(a) If $X$ is a Lindelof space, then $X$ has no more than $\exp \left(\aleph_{0}\right)$ closed sets.

(b) If $\exp \left(\aleph_{0}\right)<\exp \left(\aleph_{1}\right)$ and if $X$ has no more than $\exp \left(\aleph_{0}\right)$ closed sets, then $X$ is a Lindelöf space.

Proof. (a) is a corollary to [S, Theorem 4]. For (b), we observe that if $D$ is a discrete subspace of $X$, then the mapping $Y \rightarrow \bar{Y}$ is one-to-one on $P(D)$ and hence $D$ cannot be uncountable.

In the next three theorems, Theorems 3.3 and 3.5 will be used to obtain some characterizations of separable and hereditarily separable neighborhood $F$. spaces.

THEOREM 3.8. Let $X$ be a Lindelöf neighborhood F-space which has at most countably many isolated points. Then $X$ is separable.

Proof. Let $B$ be a neighborhood F-system for $X$. We will find a countable dense subset of $X$.

For each $m \in \mathrm{N}$ there exists a countable subset $D(m)$ of $X$ such that $X=$ $\bigcup\{$ interior of $B(m, d) \mid d \in D(m)\}$. Let $I$ be the set of isolated points of $X$, and let $D=I \cup \cup\{D(m) \mid m \in \mathbf{N}\}$. Then $D$ is countable. It is also dense.

For suppose that there exists a set $B(n, x)$ such that $B(n, x) \cap D=\varnothing$. Let $i \in \mathrm{N}$ be provided by condition (iii) of the definition of an F-system, where (iii) is applied to the point $x$ and the closed set $F=\bar{D}$. Because $x \notin I$, there is a point $y$ in the interior of $B(i, x)$ with $y \neq x$. Let $k$ be as in condition (iii), and choose $z \in\{x, y\}$ with $z \notin B(k, F)$. Then $D(k) \subset F$, so $X \subset B(k, D(k)) \subset B(k, F)$ $\subset X \backslash\{z\}$, which is a contradiction.

The space $\psi$ in Example 3.12 is a separable neighborhood $F$-space that is not Lindelöf; however, as hereditary properties for neighborhood F-spaces, separable and Lindelöf are equivalent concepts.

THEOREM 3.9. Let $X$ be a neighborhood F-space. The following are equivalent.

(a) $X$ is hereditarily Lindelöf.

(b) $X$ is hereditarily separable.

(c) $X$ is perfect and Lindelöf.

Proof. In any space, (c) implies (a).

If $X$ is hereditarily separable, then each of its discrete subspaces is countable, so by Theorem $3.5, X$ is hereditarily Lindelöf.

To see that (a) implies (b) and (c), consider any subspace $Y$ of $X$. By Theorem 2.9(a), $Y$ is a neighborhood $F$-space. Since $X$ is hereditarily Lindelöf, 
$Y$ is Lindelöf, and $Y$ has at most countably many isolated points. Thus $Y$ is separable by Theorem 3.8 .

To establish (c), we need to prove that a closed subset $F$ of $X$ is a $G_{\delta}$. Since every $F$-space is $T_{1}$, it suffices to prove that the set

$$
I=(\bigcap\{\text { interior of } B(n, F) \mid n \in \mathrm{N}\}) \backslash F
$$

is countable (where $B$ is a neighborhood F-system for $X$ ).

Suppose that $I$ is uncountable. By (a), there exists a limit point $p$ of $I$, with $p \in I$, and because each $B(n, p)$ is a neighborhood of $p$, it follows that each $B(n, p) \cap$ $(I \backslash\{p\}) \neq \varnothing$. Let $i \in \mathbf{N}$ be as in condition (iii), where (iii) is applied to the point $p$ and the closed set $F$. Next choose a point $q \in B(i, p) \cap(I \backslash\{p\})$. Then for some $k \in \mathbf{N},\{p, q\} \not \subset B(k, F)$, which contradicts the fact that $\{p, q\} \subset I$.

THEOREM 3.10. Let $X$ be a separable F-space, and assume that $\exp \left(\aleph_{1}\right)>$ $\exp \left(\aleph_{0}\right)$.

(a) If $X$ is normal, then $X$ is Lindelöf.

(b) If $X$ is completely normal, then $X$ is hereditarily Lindelöf.

Proof. In [J, Theorems 3 and 4] F. B. Jones proved that if $\exp \left(\aleph_{1}\right)>$ $\exp \left(\aleph_{0}\right)$ and if a $T_{1}$-separable space $X$ is normal (completely normal), then $X$ is $\aleph_{1}$-compact (every discrete subspace of $X$ is countable). Now apply Theorems 3.3 and 3.5.

We conclude this section with some results concerning compact $F$-spaces.

THEOREM 3.11. Every countably compact F-space is compact.

This is an immediate consequence of Theorem 3.3 and the fact that every countably compact space is $\aleph_{1}$-compact.

EXAmple 3.12. A condition known to be implied by countable compactness, equivalent to it in normal spaces, and equivalent to pseudocompactness in completely regular spaces is feeble compactness-the requirement that every locally finite family of open sets be finite. The space $\psi$ in [GJ, 5I] shows that in Theorem 3.11 countable compactness cannot be weakened to feeble compactness even for a locally compact zero-dimensional Moore space (R. W. Heath has informed the authors that several others have noticed that $\psi$ is a Moore space; e.g., see [G2]).

For symmetrizable spaces, Theorem 3.11 is due to Nedev [N1]. Furthermore, he proved the following.

THEOREM 3.13 (S. NEDEv). Every symmetrizable $\aleph_{1}$-compact space is hereditarily Lindelöf, and every Hausdorff symmetrizable countably compact space is metrizable.

The second statement of Theorem 3.13 is an immediate consequence of the first statement and the result of Arhangel'skii [A2, pp. 126-127] that every com- 
pact Hausdorff symmetrizable space is metrizable. For a proof of the first statement, based on Theorem 3.5, assume that $X$ is a symmetrizable $\aleph_{1}$-compact space with symmetric $d$ (and with $B(n, x)=\{y \mid d(x, y)<1 / n\}$ ) in which there is an uncountable discrete subspace $D$. Then for some $k \in \mathbf{N}$ there exist an uncountable $B-k$ discrete subset $Y$ of $D$ and a family $V$ of open subsets of $X$ such that each $B(k, y), y \in Y$ is contained in some member of $V$ and each member of $\checkmark$ contains exactly one point of $Y$. By Lemma 3.1, $Y$ is not closed, so there exists a point $x \in X \backslash Y$ such that $B(n, x) \cap Y \neq \varnothing$ for every $n \in \mathrm{N}$. From this it follows that $x$ is a limit point of $Y$. But if $y$ is any point of $Y$ for which $y \in$ $B(k, x)$, i.e., for which $d(x, y)<1 / k$, then $x \in B(k, y)$, so there is an open set $V \in V$ with $x \in V$ and $V \cap Y$ finite, which is impossible.

The compact Hausdorff space $E$ in Example 2.4 shows that the first statement in Theorem 3.13 does not extend to $F$-spaces, for the closed set $[0,1] \times$ $\{1\}$ is not a $G_{\delta}$. One can, however; obtain the following result (which partially answers Arhangel'skiir's question [A2] as to whether or not every weakly first countable, compact Hausdorff space is first countable).

THEOREM 3.14. Every compact Hausdorff F-space $X$ is first countable (and hence is a neighborhood F-space).

Proof. Let $p \in X$. We wish to prove that $p$ has a countable neighborhood base.

Since $X$ is compact Hausdorff, it suffices for us to prove that its subspace $Y=X \backslash\{p\}$ is Lindelöf. According to Theorem 2.9, the open subspace $Y$ is an F-space, so by Theorem 3.3 we only need to show that $Y$ is $\aleph_{1}$-compact.

Now suppose $I$ is an infinite subset of $Y$ which has no limit point in $Y$. Then $I$ is a discrete subspace of $X$ and $\bar{I}=I \cup\{p\}$ is just the one-point compactification of the space $I$. Thus $\bar{I}$ is a Fréchet space. Since $\bar{I}$ is also an $F$-space by Theorem 2.9(b), it follows from Theorem 2.6(c) that $\bar{I}$ is first countable. Thus I must be countable.

COROllary 3.15. Let $X$ be a compact Hausdorff F-space. The following are equivalent.

(a) $X$ is perfect.

(b) $X$ is hereditarily separable.

(c) $X$ is hereditarily Lindelöf.

(d) Each discrete subspace of $X$ is countable.

For arbitrary compact Hausdorff spaces, (a) $\Rightarrow(b)$ and $(d) \Rightarrow(b)$ are independent of ZFC [T] , and the one-point compactification of a space in [JKR] shows that neither $(d) \Rightarrow(c)$ nor $(b) \Rightarrow(c)$ is provable in ZFC. The next examples show that $X$ must be $T_{2}$ in 3.14 and 3.15.

EXAMPLE 3.16. Let $H$ be as in Example 2.5, and let $H^{*}$ be the one-point 
compactification of $H$. Then $H^{*}$ is not first countable, but it is shown in [FH] that $H^{*}$ is symmetrizable (and that compact subsets of $H^{*}$ are closed).

While (a), (b), and (c) hold for $H^{*}$, the compact symmetrizable space $C$ in Example 2.13 satisfies (b) and (c) but not (a).

COROllary 3.17. Let $X$ be a compact Hausdorff F-space which has at most countably many isolated points. Then $X$ is separable.

4. Perfect compact Hausdorff F-spaces. The purpose of this section is to provide examples to show that there does not exist a universal perfect compact Hausdorff F-space.

By a universal perfect compact Hausdorff space ( $F$-space) $U$ we just mean a perfect compact Hausdorff space ( $F$-space) such that every perfect compact Hausdorff space (F-space) is homeomorphic with a subspace of $U$. Thus we will prove that there is no $F$-analogue of the theorem that every compact metrizable space is homeomorphic with a subspace of the Hilbert cube.

The theorem that there exists no universal perfect compact Hausdorff space is due to Filippov [F1]. He established the result by showing that there is a family $N$ of perfect compact Hausdorff spaces such that $|N|=\exp \left(\exp \left(\aleph_{0}\right)\right)$ and no two spaces in $N$ are homeomorphic. Then he appealed to the fact that no perfect compact Hausdorff space $U$ can have more than $\exp \left(\aleph_{0}\right)$ closed subspaces.

It follows easily from proofs in $\S 6$ that the spaces in the family $N$ are not F-spaces. We will construct some spaces similar to Filippov's and use a different method to obtain the following theorem.

THEOREM 4.5. There exists a family L of perfect compact Hausdorff F. spaces such that $|L|>\exp \left(\aleph_{0}\right)$ and no two spaces in $L$ are homeomorphic. Therefore, there exists no universal perfect compact Hausdorff F-space.

Lemmas 4.1-4.4 provide a proof of Theorem 4.5.

Let $I=[0,1]$, and let $L$ and $B$ be as in Example 2.3. Then $L=I \times\{0,1\}$, and for $n \in \mathrm{N}$ and $x \in I$,

$$
\begin{aligned}
& B(n,(x, 0))=\{(x, 0)\} \cup(L \cap((x-1 / n, x) \times\{0,1\})), \\
& B(n,(x, 1))=\{(x, 1)\} \cup(L \cap((x, x+1 / n) \times\{0,1\})) .
\end{aligned}
$$

For a set $M \subset I$, denote by $L(M)$ the quotient space of $L$ obtained by identifying, for each $m \in M$, the points $(m, 0)$ and $(m, 1)$. The natural mapping of $L$ onto $L(M)$ will be denoted by $p_{M}$ or, if no confusion is possible, just $p$. Likewise, the natural mapping of $L(M)$ onto $I$ will be denoted by $q_{M}$ or $q$. Thus $q$ identifies, for each $x \in I \backslash M$, the points $p((x, 0))$ and $p((x, 1))$. We define $B^{\prime}: \mathbf{N} \times L(M)$ $\rightarrow P(L(M))$ as follows. For each $n \in N, x \in I$, and $s \in\{0,1\}$,

$$
B^{\prime}(n, p((x, s)))=\bigcup\{p(B(n,(x, t))) \mid(x, t) \in p((x, s))\} .
$$


Let us make some observations about $L(M)$.

LEMMA 4.1. (a) $B^{\prime}$ is a neighborhood F-system for the space $L(M)$. Moreover, each $B^{\prime}(n, p((x, s)))$ is an open set.

(b) $L(M)$ is compact, hereditarily Lindelöf, and Hausdorff. Thus $p$ is a closed mapping.

(c) The subspace $p(M \times\{0,1\})$ of $L(M)$ is homeomorphic with the subspace $M$ of $I$.

(d) The mapping $p$ carries $L \backslash(M \times\{0,1\})$ homeomorphically onto $L(M) \backslash p(M \times\{0,1\})$.

(e) No uncountable subspace of $L(M) \backslash p(M \times\{0,1\})$ is metrizable.

Proof. (a) For $x \in I M$, each $p^{-1}\left(B^{\prime}(n, p((x, s)))\right)=B(n,(x, s))$, and for $x \in M, p^{-1}\left(B^{\prime}(n, p((x, s)))\right)=B(n,(x, 0)) \cup B(n,(x, 1))$. Thus each $B^{\prime}(n, p((x, s)))$ is open in $L(M)$. The proof that $B^{\prime}$ is an F-system is similar to the one needed in Example 2.3.

(b) Since $p: L \rightarrow L(M)$ is continuous, $L(M)$ is compact and hereditarily Lindelöf.

For $x \in I M$, each $B^{\prime}(n, p((x, 0))) \cap B^{\prime}(n, p((x, 1)))=\varnothing$, and for $x, y \in I$, $s, t \in\{0,1\}$, and $m \in \mathrm{N}$ with $1 / m \leqslant|x-y|$, we have $B^{\prime}(2 m, p((x, s))) \cap$ $B^{\prime}(2 m, p((y, t)))=\varnothing$. Thus $L(M)$ is Hausdorff, and $p$ is a closed mapping.

(c) The mapping $q: L(M) \rightarrow I$ is closed, and since $q \mid p(M \times\{0,1\})$ is oneto-one and $p(M \times\{0,1\})=q^{-1}(M)$, it follows that (c) holds. Similarly, one obtains (d).

(e) By (d), an uncountable subspace of $L(M) \backslash p(M \times\{0,1\})$ is homeomorphic with an uncountable subspace $Z$ of $L \backslash(M \times\{0,1\})$, and for some $s \in$ $\{0,1\}, Y=Z \cap(I \times\{s\})$ is uncountable. Clearly, $Y$ is homeomorphic with an uncountable subspace of the Sorgenfrey line $S$. The argument given in the last paragraph of $\$ 2$ shows that no uncountable subspace of $S$ is metrizable. Thus $Z$ cannot be metrizable.

LEMma 4.2. Suppose that $M$ and $M^{\prime}$ are subsets of $I$ such that $L(M)$ and $L\left(M^{\prime}\right)$ are homeomorphic, and $|M|=\left|M^{\prime}\right|=\exp \left(\aleph_{0}\right)$. Then there exists a continuous mapping $f: L(M) \rightarrow I$ such that $\left|f\left(p_{M}(M \times\{0,1\})\right)\right|=\exp \left(\aleph_{0}\right)$ and $f\left(p_{M}(M \times\{0,1\})\right) \backslash M^{\prime}$ is countable.

Proof. Let $h$ be a homeomorphism of $L(M)$ onto $L\left(M^{\prime}\right)$. By Lemma 4.1(c), $h\left(p_{M}(M \times\{0,1\})\right)$ is metrizable, so applying Lemma $4.1(\mathrm{e})$ to the space $L\left(M^{\prime}\right)$, one sees that $h\left(p_{M}(M \times\{0,1\})\right) \backslash p_{M^{\prime}}\left(M^{\prime} \times\{0,1\}\right)$ is countable. The mapping $f=q_{M^{\prime}} \circ h$ thus has the desired properties.

LEMMA 4.3 ([GJ, 12B]). There exists a family $M$ of subsets of I such that 
$|M|>\exp \left(\aleph_{0}\right),|M|=\exp \left(\aleph_{0}\right)$, and $\left|M \cap M^{\prime}\right|<\exp \left(\aleph_{0}\right)$ whenever $M, M^{\prime} \in M$ with $M \neq M^{\prime}$.

LEMMA 4.4. Let $M \in M$, and let $E=\{E \in M \mid L(M)$ and $L(E)$ are homeomorphic\}. Then $|E| \leqslant \exp \left(\aleph_{0}\right)$.

Proof. Let $C=\{f: L(M) \rightarrow I \mid f$ is continuous $\}$, and note that because $L(M)$ is separable, $|C| \leqslant \exp \left(\aleph_{0}\right)$. For each $E \in E$ there exists by Lemma 4.2 an $f_{E} \in C$ such that $\left|f_{E}\left(p_{M}(M \times\{0,1\})\right)\right|=\exp \left(\aleph_{0}\right)$ and $f_{E}\left(p_{M}(M \times\{0,1\})\right) \backslash E$ is countable. Now for any $E, E^{\prime} \in E$, if $f_{E}=f_{E^{\prime}}$, then it follows that the set $E \cap E^{\prime}$ has $\left|E \cap E^{\prime}\right| \geqslant \exp \left(\aleph_{0}\right)$, but since $E \subset M$, the latter implies that $E=E^{\prime}$. Thus the mapping $E \rightarrow f_{E}$ is one-to-one and $|E| \leqslant|C|$.

Proof OF Theorem 4.5. To complete the proof, one now proceeds as in [F1]. Let $L$ be a family of spaces in $\{L(M) \mid M \in M\}$ obtained by selecting one member from each homeomorphism class. Since $|M|>\exp \left(\aleph_{0}\right)$, it follows from Lemma 4.4 that $|L|>\exp \left(\aleph_{0}\right)$.

5. A separable compact Hausdorff $\dot{r}$-space that is not hereditarily separable. An example will be given to show that Theorems 3.8 and 3.9 cover distinct families and also to show that the first statement in Theorem 3.13 does not hold for all separable $F$-spaces.

In the following, $J$ denotes the open interval $(0,1), D$ is the discrete space $\{0,1,2\}$, and $K$ is the family of all nondecreasing mappings of $J$ into $D$, with the topology of pointwise convergence. Thus $K$ has the topology it inherits as a closed subspace of the product space $P=\{f \mid f: J \rightarrow D\}$, and so $K$ is compact and Hausdorff. We will be interested in a certain subspace $M$ of $K$. Let us indicate which points of $K$ are in $M$ and how they will be denoted.

For $i \in\{0,2\}, c_{i}$ is the constant function in $K$ whose value is $i$. For $t \in$ $J: a^{t}, a_{t}$ denote the $\{0,2\}$ valued functions in $K$ such that for each $x \in J, a^{t}(x)=$ 2 if and only if $x \geqslant t$, and $a_{t}(x)=0$ if and only if $x \leqslant t$; and the symbol at denotes the function in $K$ such that for each $x \in J$, at $(x)=1$ if and only if $x=t$. If $k, n \in \mathbf{N}$ with $1<k<2^{n}$, then $a k n$ is the function in $K$ defined by the rule $a k n(x)=1$ if and only if $x \in\left[(k-1) / 2^{n}, k / 2^{n}\right]$. Let $M$ be the set of all such functions $c_{i}, a^{t}, a_{t}, a t$, and $a k n$, with the subspace topology.

THEOREM 5.4. The space $M$ is a separable compact Hausdorff F-space in which there is a discrete subspace of cardinality $\exp \left(\aleph_{0}\right)$.

The proof of Theorem 5.4 is provided by Lemmas 5.1-5.3.

LEMmA 5.1. (a) The set $Y=\left\{a k n \mid k, n \in \mathrm{N}, 1<k<2^{n}\right\}$ is a countable dense subset of $M$.

(b) $\{a t \mid t \in J\}$ is a discrete subspace of $M$ having cardinality $\exp \left(\aleph_{0}\right)$.

The proof is straightforward. 
Lemma 5.2. (a) The set $T=M \backslash Y$, where $Y$ is as above, is compact.

(b) $M$ is compact.

Proof. (a) It is easy to see that $V=\left\{f \in K|| f^{-1}(1) \mid \geqslant 2\right\}$ is an open subset of the compact space $K$ and that $T=K \backslash V$.

(b) Since $M$ is the union of the compact set $T$ and the countable set $Y$, $M$ is a Lindelöf space. Thus, to prove that $M$ is compact, it suffices to prove that an infinite subset $F$ of $Y$ has a limit point in $T$.

Let us first observe that

(\#) for any $e>0,\left\{f \in Y \mid\right.$ (length of $\left.\left.f^{-1}(1)\right) \geqslant e\right\}$ is finite.

If there exists $t \in J$ with the set $Z_{t}=\{f \in F \mid f(t)=1\}$ infinite, then it follows from (\#) that the function at is a limit point of $Z_{t}$ and hence of $F$. If not, then for each $f \in F$ choose $t(f) \in f^{-1}(1)$, and let $C=\{t(f) \mid f \in F\}$. Now $C$ is infinite and has a limit point $p \in[0,1]$. If $p=0(p=1)$ then by $(\#), c_{2}\left(c_{0}\right)$ is a limit point of $F$. If $p \in J$ and $p$ is a limit point of $C \cap[p, 1)(C \cap(0, p])$, then one can show that $a_{p}\left(a^{p}\right)$ is a limit point of $F$.

A neighborhood $F$-system $B$ for $M$ will now be defined. Let $n \in \mathrm{N}$ and $f \in M$. Then $B(n, f)$ is the set of all functions $g \in M$ such that:

(a) for each $k \in \mathrm{N}$, if $1 \leqslant k<2^{n}$, then $g\left(k / 2^{n}\right)=f\left(k / 2^{n}\right)$;

(b) for each $x \in J$, if $x$ is a point of discontinuity of $f$, then $g(x)=f(x)$; and

(c) if $g \neq f$, then $g \notin\left\{a p q \mid p, q \in \mathbf{N}, q \leqslant n\right.$, and $\left.1<p<2^{q}\right\}$.

LEMMA 5.3. The mapping $B$ is a neighborhood F-system for the space $M$.

Proof. Clearly, for $f \in M$, each $B(n+1, f) \subset B(n, f)$, and $\{f\}=$ $\bigcap\{B(n, f) \mid n \in \mathrm{N}\}$. Furthermore, each $B(n, f)$ is an open-and-closed subset of $M$, for $A=\left\{a p q \in M \backslash\{f\} \mid p, q \in \mathrm{N}, q \leqslant n\right.$, and $\left.1<p<2^{q}\right\}$ is a finite set of isolated points of $M$, and $B(n, f)$ is the intersection of $M \backslash A$ with the following open-and-closed set of the product space $P$

$$
\bigcap\left\{\pi_{t}^{-1}(\{f(t)\})\right\},
$$

where $t$ ranges over the finite set $\left\{k / 2^{n} \mid 1 \leqslant k<2^{n}, k \in \mathbf{N}\right\} \cup\{t \mid f$ has a discontinuity at $t$. Since the space $M$ is compact by Lemma 5.2, any filter base of open-and-closed sets whose intersection is one point is a neighborhood base for the point. Thus (i) and (ii) hold.

To show that (iii) is satisfied, it suffices to prove that for $f \in M, n \in \mathrm{N}$, and the closed set $F=M \backslash B(n, f)$, there exists $i \in \mathrm{N}$ for which the conclusion of (iii) holds. Consider the different possibilities for $f$, where $j \in\{0,2\}$ and $t \in J$ :
(1) $f \in Y$;
(2) $f=c_{j}$;
(3) $f=a t$;
(4) $f=a_{t}$; and
(5) $f=a^{t}$

(1) In this case, it follows from (b) and (c) of the definition of $B$ that if $f=a p q$, then $B(q, f)=\{f\}$. Thus (iii) holds vacuously. 
(2) Since $f=c_{j}$ has no discontinuities, if a function $g \in M$ is in $F$, i.e., if $g$ fails to be in $B(n, f)$, then either $g \in Y$ or there must exist $1 \leqslant k^{\prime}<2^{n}$ with $g\left(k^{\prime} / 2^{n}\right) \neq f\left(k^{\prime} / 2^{n}\right)$. In either case, we obtain $f \notin B(n, g)$. Thus (iii) is satisfied if $k=i=n$.

(3) Here $f=a t$ and $t \in J$, so we may select an $i \in \mathbf{N}$ such that $i \geqslant n$, and for some $k^{\prime}, k^{\prime \prime} \in \mathrm{N}, k^{\prime} / 2^{i}<t<k^{\prime \prime} / 2^{i}<1$. Now note that for every $h \in B(i, f)$ the following hold: by (a) and (b), $h$ must map $J$ onto $D$; and by (b), $h \neq a t^{\prime}$ for any $t^{\prime} \neq t$. So $B(i, f) \backslash\{f\} \subset Y$.

Consider an arbitrary function $a p q \in B(i, f) \backslash\{f\}$. By (c), for every $g \in M \backslash\{a p q\}, a p q \notin B(q, g)$. Hence $a p q \notin B(q, F)$, and (iii) is satisfied for $k=q$.

Since cases (4) and (5) are similar, we will just show that (iii) is satisfied for a function $f=a_{t}$.

(4) Choose $i \in \mathrm{N}$ so large that $i \geqslant n$.

Let $h \in B(i, f) \backslash\{f\}$. Then whatever type of function $h$ is, one can at least conclude that for some $t^{\prime}>t, h\left(t^{\prime}\right)=0$. Choose $m \in \mathrm{N}$ for which there exists $k^{\prime} \in \mathrm{N}$ with $t<k^{\prime} / 2^{m} \leqslant t^{\prime}$.

Let $g \in F$. If $g$ fails to be in $B(n, f)$ because of $(\mathrm{a})$, then $h \notin B(n, g)$. If $g$ fails to satisfy (b), then $h \notin B(m, g)$. If $g \in\{a p q \mid q \leqslant n\}$, then $h \notin B(n, g)$. Thus we may take $k=\max \{m, n\}$, and that completes the proof.

REMARK 5.5. Several of the examples given in previous sections are ordered spaces. The space $M$ is not, for it is known that no separable completely normal $T_{1}$-space can have a discrete subspace of cardinality $\exp \left(\aleph_{0}\right)$ (see [J, Theorem 2]).

6. Products of F-spaces. Semimetrizability is known to be countably productive. We will consider some suitable restrictions under which symmetrizability is countably productive, and also under which necessary and sufficient conditions can be given that a product space be an F-space. In view of the following result, we will only consider products of countably many factor spaces.

THEOREM 6.1 (J. NovÁK [N3]). Let $X=\Pi\left\{X_{a} \mid a \in A\right\}$, where each $X_{a}$ is a $T_{1}$-space, and suppose that $X$ is a sequential space. Then the set $C=$ $\left\{a \in A|| X_{a} \mid>1\right\}$ is countable.

A short proof can be obtained as follows: Suppose that $C$ is uncountable. Then the product space $P=\left\{f \mid f: \aleph_{1} \rightarrow\{0,1\}\right\}$, where $\{0,1\}$ has the discrete topology, is homeomorphic with a closed subspace of $X$, and so $P$ must be a sequential space. But $D=\left\{f \in P \mid f^{-1}(1)\right.$ is countable $\}$ is a sequentially closed, proper dense subset of $P$.

Notation. In a product space $X=\Pi X_{n}$, the projection mapping of $X$ onto $X_{n}$ will be denoted by $\pi_{n}$, and for a point $x \in X, x(n)$ will be denoted by $x_{n}$. For a sequence $s$ of points of $X$, we will write $s^{n}$ for $s(n)$. An F-system on $X_{n}$ will be denoted by $B^{n}$. 
LEMmA 6.2. Let $X=\Pi\left\{X_{n} \mid n \in \mathrm{N}\right\}$, where for each $n \in \mathbf{N}, B^{n}$ is an $F$ system for $X_{n}$ that satisfies (*). For $x \in X$ and $i \in \mathrm{N}$, let

$$
B(i, x)=\bigcap\left\{\pi_{j}^{-1}\left(B^{j}\left(i, x_{j}\right)\right) \mid j \in \mathrm{N} \text { and } j \leqslant i\right\},
$$

and suppose that $Y$ is a sequential subspace of $X$. Then $B_{Y}$ satisfies (*) and conditions (i) and (ii) of the definition of an F-system.

Proof. Obviously $B_{Y}$ satisfies (i).

Now a sequence $\left\{x^{n}\right\}$ in $X$ converges to a point $x \in X$ if and only if each $x_{i}^{n} \rightarrow x_{i}$, and similarly, one can show that $\left\{x^{n}\right\} B$-converges to $x$ if and only if each $\left\{x_{i}^{n}\right\} \quad B^{i}$-converges to $x_{i}$. Since each $B^{i}$ satisfies (*), it thus follows that $B_{Y}$ also satisfies (*).

Because each $B^{i}$ satisfies (ii), it is easy to see that for every basic open set $V$ of $X$ and point $y \in V \cap Y$, there exists $i \in \mathrm{N}$ with $B_{Y}(i, y) \subset V \cap Y$.

Let $F$ be a subset of $Y$ such that for each $y \in Y \backslash F$ there exists $n \in \mathrm{N}$ with $B_{Y}(n, y) \cap F=\varnothing$. Then no sequence in $F$ can $B$-converge to a point in $Y \backslash F$, and since $B_{Y}$ satisfies (*), it follows that no sequence in $F$ can converge to a point in $Y \backslash F$. Thus $F$ is a sequentially closed and hence closed subset of $Y$.

THEOREM 6.3. Let $X=\Pi\left\{X_{n} \mid n \in \mathrm{N}\right\}$, where each space $X_{n}$ admits a symmetric satisfying (*). The following are equivalent.

(a) $X$ is a sequential space.

(b) $X$ is symmetrizable.

Proof. Every $F$-space is sequential. Conversely, suppose that each $X_{n}$ has an F-system $B^{n}$ satisfying (*) and (iii)'. Let $B$ be as in Lemma 6.2. Then obviously $B$ satisfies (iii)', so $X$ is symmetrizable via $B$.

Next we obtain a useful characterization of symmetrizable spaces (which may be known). First a definition is needed.

We will say that an $F$-system $B$ for a space $X$ satisfies (iii)" provided that for each closed set $F \subset X$ and for each point $x \in X \backslash F$, there exists $k \in \mathrm{N}$ such that $x \notin B(k, F)$.

LEMMA 6.4. Let $X$ be a topological space, and suppose that there exists a mapping $B: \mathrm{N} \times X \rightarrow P(X)$ satisfying conditions (i), (ii), and (iii)". Then $X$ is symmetrizable.

Proof. For $x \in X$, let $d(x, x)=0$, and for $y \in X \backslash\{x\}$, let

$$
d(x, y)=1 / \min \{n \mid x \notin B(n, y) \text { and } y \notin B(n, x)\} .
$$

Then $d(x, y) \geqslant 0, d(x, y)=d(y, x)$, and $d(x, y)=0$ if and only if $x=y$.

Let $F$ be a closed set and $x \in X \backslash F$. We must show that $d(x, F)>0$. By hypothesis, there exists $k \in \mathrm{N}$ such that $x \notin B(k, F)$. Choose $m \in \mathrm{N}$ so that $m \geqslant k$ and $B(m, x) \cap F=\varnothing$. Then $d(x, F) \geqslant 1 / m>0$. 
Conversely, let $G \subset X$, and suppose that for each $x \in X \backslash G$ there is a $k(x)$ $\in \mathrm{N}$ with $d(x, G) \geqslant 1 / k(x)$. Then $X \backslash G=\bigcup\{B(k(x), x) \mid x \in X \backslash G\}$, so $G$ is a closed subset of $X$.

REMARK 6.5. It is interesting to observe that in Lemma 6.4 one can replace the requirement that $B$ satisfy (iii)" by the requirement that there exist a countable set $C \subset X$ such that for each closed set $F \subset X$ and $x \in X \backslash(C \cup F), x \notin$ $\bigcap\{B(k, F) \mid k \in \mathrm{N}\}$. For, if $C=\left\{c_{i} \mid i \in \mathrm{N}\right\}$ and $B^{\prime}(n, x)=\{x\} \cup$ $\left(B(n, x) \backslash\left\{c_{i} \mid i \leqslant n\right\}\right)$, then $B^{\prime}$ satisfies (i), (ii), and (iii)". Thus, for example, a countable, weakly first countable $T_{1}$-space is symmetrizable.

LEMma 6.6. Let $X$ be a topological space, and suppose that, for each $j \in \mathbf{N}$, $B^{j}$ is an F-system for $X$ satisfying (*). If $X$ is not symmetrizable, then there exist a closed set $F \subset X$ and a point $x \in X \backslash F$ such that $x \in \bigcap\left\{B^{\prime}(k, F) \mid j, k \in \mathrm{N}\right\}$.

Proof. Let $P=\{f \mid f: \mathrm{N} \rightarrow X\}$, with the product topology, let $B$ be as in Lemma 6.2, and denote the set of constant functions in $P$ by $Y$. Then $Y$ is homeomorphic with $X$, so $Y$ is a sequential space, and $B_{Y}$ is an F-system for $Y$ by Lemma 6.2 .

For $x \in X$ and $n \in \mathrm{N}$, define $B^{\prime}(n, x)=\bigcap\left\{B^{j}(n, x) \mid j \in \mathrm{N}, j \leqslant n\right\}$ and note that each $B^{\prime}(n, x)=\pi_{1}\left(B_{Y}(n,\{x\})\right)$. Thus $B^{\prime}$ is an F-system for $X$, so by Lemma 6.4 there exist a closed set $F \subset X$ and a point $x \in X \backslash F$ such that $x \in$ $\bigcap\{B(k, F) \mid k \in \mathbf{N}\}$.

Let us show that $x \in \bigcap\left\{B^{j}(k, F) \mid j, k \in \mathrm{N}\right\}$. If $j, k \in \mathrm{N}$ and $j<k$, then $x \in B^{\prime}(k, F) \subset B^{j}(k, F)$. If $j, k \in \mathrm{N}$ and $j>k$, then $x \in B^{\prime}(j, F) \subset B^{j}(j, F) \subset$ $B^{j}(k, F)$.

THEOREM 6.7. Let $X$ be a topological space, and let $T=\{0\} \cup\{1 / n \mid n \in N\}$ have its usual topology. If the product space $X \times T$ admits an F-system satisfying (*), then the space $X$ is symmetrizable.

Proof. Let us assume that $X$ is not symmetrizable but that $X \times T$ admits such an $F$-system $B$.

For each $t \in T, Y_{t}=X \times\{t\}$ is a closed subspace of $X \times T$, so $B$ induces on $Y_{t}$ an $F$-system satisfying (*). Then

$$
B^{t}(n, x)=\pi_{1}\left(B_{Y_{t}}(n,(x, t))\right)
$$

is an F-system for $X$ that satisfies (*).

Since $T$ is countable, Lemma 6.6 implies that there exist a closed set $F \subset$ $X$ and a point $x \in X \backslash F$ such that $x \in \bigcap\left\{B^{t}(k, F) \mid t \in T, k \in \mathrm{N}\right\}$. From this it follows that, for every $t \in T$,

$$
(x, t) \in \bigcap\{B(k, F \times\{t\}) \mid k \in \mathrm{N}\} .
$$

Let us now consider the closed set $F \times T$ and the point $(x, 0) \in$ 
$(X \times T) \backslash(F \times T)$. Since $B$ is an F-system for $X \times T$, we may let $i$ be as in condition (iii). Then $(x, 1 / n) \rightarrow(x, 0)$, and $B$ satisfies (*), so for some $n$ $\in \mathrm{N},(x, 1 / n) \in B(i,(x, 0))$. By (iii), there is a $k \in \mathrm{N}$ with $\{(x, 0),(x, 1 / n)\} \not$ $B(k, F \times T)$, but by (\#), $\{(x, 0),(x, 1 / n)\} \subset B(k, F \times\{0\}) \cup B(k, F \times\{1 / n\})$, so we have a contradiction.

Corollary 6.8. Let $X$ be a Hausdorff space, and let $T$ be as above. The following are equivalent.

(a) $X \times T$ is an F-space.

(b) $X$ is symmetrizable.

(c) $X \times T$ is symmetrizable.

Proof. By Theorem 2.6, any $F$-system for $X \times T$ must satisfy (*), so (a) implies (b). That (b) implies (c) is a consequence of Theorem 6.3 and the wellknown fact that the product of two Hausdorff sequential spaces, one of which is locally compact, is a sequential space.

Corollary 6.9. A Hausdorff F-space $X$ is symmetrizable if it is a product of two nondiscrete spaces.

Proof. Suppose that $X=Y \times Z$, and neither $Y$ nor $Z$ is a discrete space.

Since $Z$ is homeomorphic with a closed subspace of $X, Z$ is a sequential space. Thus there exists a subset of $Z$ which is not sequentially closed, and from this it follows that the Hausdorff space $Z$ has a closed subspace $T$ homeomorphic with $T$. Then $Y \times T^{\prime}$ is closed in $X$ and hence is an $F$-space, so by Corollary $6.8, Y$ is symmetrizable. Similarly, one proves that $Z$ is symmetrizable. Now $X=Y \times Z$ is a sequential space which is a product of symmetrizable spaces, so by Theorem $6.3, X$ is symmetrizable.

We will conclude the paper by showing that there exists a regular Lindelöf symmetrizable space whose product with $\mathbf{Q}$ is not symmetrizable. (The result that there exists a sequential space, namely $Q^{\prime}=$ the quotient space of $\mathbf{Q}$ obtained by identifying the integers, such that $Q^{\prime} \times Q$ is not a sequential space, is due to S. P. Franklin [F2, Example 1.11]; however, the space $Q^{\prime}$ cannot be used here, for one can show that $Q^{\prime}$ is not weakly first countable.) We will also obtain a symmetrizable space analogue of the well-known Boehme-Michael theorem [B1], [M] that a regular $T_{1}$ sequential space $X$ is locally countably compact if and only if for every sequential space $Y, X \times Y$ is a sequential space (a similar theorem concerning $\Omega$-net spaces is obtained in [G1]).

Notation. In the lemmas below, $W$ will denote the set $\{0\} \cup N \cup$ $\{m+1 / n \mid m, n \in \mathbf{N}\}$, with the topology it inherits from the space $H$ in Example 2.5. Note that $W$ is a closed subset of $H$ and hence is a regular Lindelöf symmetrizable space. 
LEMMA 6.10. Let $R$ be a first countable Hausdorff space for which $P=$ $W \times R$ is a sequential space. Then $R$ is regular and locally countably compact.

Proof. Let $p \in R$ and let $\left\{V_{m} \mid m \in N\right\}$ be a neighborhood base for $p$ such that each $V_{m} \supset V_{m+1}$.

If there is a neighborhood $V$ of $p$ which contains no closed neighborhood of $p$, then for each $m \in \mathrm{N}$ there is a sequence $\left\{y_{m n} \mid n \in \mathrm{N}\right\}$ in $V_{m} \backslash\{p\}$ which converges to a point $q_{m}$ of $R \backslash V$. In this case define

$$
A=\left\{\left(m+1 / n, y_{m n}\right) \mid m, n \in \mathrm{N}, n \geqslant 2\right\} \cup\left\{\left(m, q_{m}\right) \mid m \in \mathrm{N}\right\} .
$$

If, instead, every neighborhood of $p$ contains a closed neighborhood of $p$, and no neighborhood of $p$ is countably compact, then for each $m$ there is a sequence $\left\{y_{m n} \mid n \in N\right\}$ in $V_{m} \backslash\{p\}$ having no cluster point in $X$, and one can define

$$
A=\left\{\left(m+1 / n, y_{m n}\right) \mid m, n \in \mathrm{N}, n \geqslant 2\right\} .
$$

In either case, the point $(0, p) \in \bar{A} \backslash A$, so $A$ is not a closed subset of $P$. On the other hand, $A$ is clearly a closed subset of $P \backslash\{(0, p)\}$, so if $P$ were a sequential space, then there would exist a sequence $\left\{a^{n}\right\}$ in $A$ with $a^{n} \rightarrow(0, p)$. Because (in the first case) $p$ has a neighborhood $V$ with $V \cap\left\{q_{m} \mid m \in N\right\}=\varnothing$, it would follow from the relation $a_{2}^{n} \rightarrow p$ that in either case $\left\{a_{1}^{n}\right\}$ is eventually in $M Z$, in contradiction of the fact (noted in Example 2.7) that no sequence in $H \backslash \mathbf{Z}$ can converge to 0 .

The next lemma is to be compared with [F3, Proposition 7.3].

A point $x$ of a space $X$ will be called a regular $G_{\delta}$ if there exists a sequence $\left\{V_{m} \mid m \in \mathbf{N}\right\}$ of neighborhoods of $x$ with $\{x\}=\left\{\mathrm{Cl}_{X} V_{m} \mid m \in \mathrm{N}\right\}$.

LEMMA 6.11. Let $X$ be a symmetrizable space in which each point is a regular $G_{\delta}$. Then the following are equivalent.

(a) $X$ is first countable.

(b) $W$ cannot be embedded homeomorphically in $X$ as a closed subset.

Proof. Because 0 does not have a countable neighborhood base in $W$, (a) implies (b).

Conversely, suppose there is a point $x \in X$ which has no countable neighborhood base. Let $d$ be a symmetric for the space $X$. Then for some $e>0, x$ is not in the interior of $S=\{y \in X \mid d(x, y)<e\}$. Let $F=\{y \in X \mid d(y, X \backslash S)=0\}$. Since $x \notin F, F$ cannot be a closed set, for, otherwise, $X \backslash F$ would be an open set with $x \in X \backslash F \subset S$. Thus there is a point $z \in X \backslash F$ with $d(z, F)=0$. Because $z$ is a regular $G_{\delta}$, one can find a subset of $F \cap S$, indexed in a one-to-one way as $\left\{f_{m} \mid m \in N\right\}$, and a decreasing sequence $\left\{V_{m} \mid m \in N\right\}$ of open neighborhoods of $z$ such that $\{z\}=\bigcap\left\{\mathrm{Cl} V_{m} \mid m \in \mathrm{N}\right\}$ and for each $m, d\left(z, f_{m}\right)<1 / m$ and $f_{m} \in$ $V_{m} \backslash \mathrm{Cl} V_{m+1}$. From this and the relations $f_{m} \in S$ and $d\left(f_{m}, X \backslash S\right)=0$, it fol- 
lows that for each $m$ there is a subset of $V_{m} \backslash\left(\mathrm{Cl} V_{m+1} \cup S\right)$, indexed in a oneto-one way as $\left\{c_{m n} \mid n \in \mathrm{N}, n \geqslant 2\right\}$, such that $d\left(f_{m}, c_{m n}\right)<1 / n$.

Let

$$
C=\{z\} \cup\left\{f_{m} \mid m \in \mathrm{N}\right\} \cup\left\{c_{m n} \mid m, n \in \mathrm{N}, n \geqslant 2\right\} .
$$

We will prove that $C$ is a closed subset of $X$ which, with the subspace topology, is homeomorphic with $W$.

To see that $C$ is closed, it suffices to observe that for each $m, K_{m}=$ $\left\{f_{i} \mid i \leqslant m\right\} \cup\left\{c_{i n} \mid i \leqslant m\right\}$ is a compact subset of the Hausdorff space $X$, and so

$$
C=\bigcap\left\{K_{m} \cup \mathrm{Cl} V_{m+1} \mid m \in \mathrm{N}\right\}
$$

is an intersection of closed sets.

Now consider the subspace topology on $C$. Because the sets $V_{m} \backslash \mathrm{Cl} V_{m+1}$, $m \in N$, are pairwise disjoint, each set $\left\{f_{m}\right\} \cup\left\{c_{m n} \mid n \geqslant 2\right\}$ is a clopen subset of $C$ which is homeomorphic with the clopen subspace $\{m\} \cup\{m+1 / n \mid n \geqslant 2\}$ of the space $W$. Since $d\left(z, f_{m}\right)<1 / m$ and $d \mid C \times C$ is a symmetric for the subspace topology on the closed set $C$, every neighborhood $V$ of $z$ in $C$ contains a set of the form $U=\{z\} \cup\left\{f_{m} \mid m \geqslant k\right\} \cup\left\{c_{m n} \mid m \geqslant k, n \geqslant q(m)\right\}$.

Conversely, every set of the form $U$ must be open in $C$, for $U \supset$ $\{y \in C \mid d(z, y)<\min \{1 / k, d(z, X / S)\}\}$, and obviously for each point $x \in U \backslash\{z\}$ there is a $g>0$ with $U \supset\{y \in C \mid d(x, y)<g\}$. Thus $C$ is homeomorphic with $W$.

THEOREM 6.12. Let $X$ be a symmetrizable space in which every point is a regular $G_{\delta}$. The following are equivalent.

(a) For each space $Y$, if $Y$ is symmetrizable via a symmetric satisfying (*) (e.g., if $Y$ is Hausdorff), then $X \times Y$ is symmetrizable.

(b) $X \times W$ and $X \times Q$ are symmetrizable.

(c) $X$ is locally compact.

Proof. Obviously (a) implies (b).

Let us suppose that (b) is true. If $X$ fails to be first countable, then by Lemma 6.11 there is a closed subset $C$ of $X$ such that $C$ is homeomorphic with $W$. But then $C \times \mathbf{Q}$ is a closed subset of the sequential space $X \times \mathbf{Q}$ and hence must be sequential, whereas according to Lemma $6.10, C \times Q$ is not a sequential space. Thus $X$ is first countable and Hausdorff, and so by Lemma 6.10 it is also regular and locally countably compact. Hence (c) holds.

We recall that in a $T_{1}$ sequential space countable compactness implies sequential compactness by a result of S. P. Franklin, and by a theorem of Boehme the product of a sequential space and a regular, locally sequentially compact sequential space is a sequential space. Therefore, (c) implies (a).

\section{BIBLIOGRAPHY}

[A1] R. Arens, Note on convergence in topology, Math. Mag. 23 (1950), 229-234. MR 12, 271. 
[A2] A. V. Arhangel'skiī, Mappings and spaces, Uspehi Mat. Nauk 21 (1966), no. 4 (130), 133-184 = Russian Math. Surveys 21 (1966), no. 4, 115-162. MR 37 \#3534.

[AU] P. Aleksandrov and P. Urysohn, 'On compact topological spaces,' in P. S. Urysohn, Works in topology and other fields of mathematics. Vol. 2, GITTL, Moscow, 1951 , pp. 848-963. MR 14, 122.

[B1] T. K. Boehme, Linear s-spaces, Symposium on Convergence Structures, University of Oklahoma, 1965.

[B2] D. A. Bonnett, A symmetrizable space that is not perfect, Proc. Amer. Math. Soc. 34 (1972), 560-564. MR 45 \#4343.

[FH] G. D. Faulkner and P. W. Harley III, Metrization of symmetric spaces, Canad. J. Math. 27 (1975).

[F1] V. V. Filippov, On perfectly normal bicompacta, Dokl. Akad. Nauk SSSR (1969), 736-739 = Soviet Math. Dokl. 10 (1969), 1503-1507. MR 41 \#1006.

[F2] S. P. Franklin, Spaces in which sequences suffice, Fund. Math. 57 (1965), 107115. MR 31 \#5184.

[F3] - Spaces in which sequences suffice, II, Fund. Math. 61 (1967), 51-56. MR 36 \#5882.

[GJ] L. Gillman and M. Jerison, Rings of continuous functions, University Series in Higher Math., Van Nostrand, Princeton, N. J., 1960. MR 22 \#6994.

[G1] J. W. Goldston, Topologies determined by classes of nets, Doctoral Dissertation, University of North Carolina, Chapel Hill, N. C., 1975.

[G2] J. W. Green, Moore-closed spaces, completeness and centered bases, General Topology and Appl. 4 (1974), 297-313.

[H] R. W. Heath, Semi-metric spaces and related spaces, Proc. Arizona State University Topology Conference, 1967.

[HM] R. W. Heath and E. A. Michael, A property of the Sorgenfrey line, Compositio Math. 23 (1971), 185-188. MR 44 \#419.

[J] F. B. Jones, Concerning normal and completely normal spaces, Bull. Amer. Math. Soc. 43 (1937), 671-677.

[JKR] I. Juhasz, K. Kunen and M. E. Rudin, Two more hereditarily separable nonLindelöf spaces (to appear).

[M] E. Michael, Local compactness and Cartesian products of quotient maps and k-spaces, Ann. Inst. Fourier (Grenoble) 18 (1968), fasc. 2, 281-286. MR 39 \#6256.

[N1] S. I. Nedev, Symmetrizable spaces and final compactness, Dokl. Akad. Nauk SSSR 175 (1967), 532-534 = Soviet Math. Dokl. 8 (1967), 890-892. MR 35 \#7293.

[N2] V. Niemytzki, Über die Axiome des metrischen Raumes, Math. Ann. 101 (1931), 666-671.

[N3] J. Novák, Sur les espaces ( $L$ ) et sur les produits cartésiens (L), Publ. Fac. Sci. Univ. Massaryk 1939, no. 273. MR 1, 221.

[O] A. J. Ostaszewski, On countably compact, perfectly normal spaces, J. Iondon Math. Soc. (to appear).

[S] R. M. Stephenson, Jr., Discrete subsets of perfectly normal spaces, Proc. Amer. Math. Soc. 34 (1972), 605-608. MR 45 \#5944.

[T] F. D. Tall, An alternative to the continuum hypothesis and its uses in general topology (to appear).

[V] J. E. Vaughan, Lexicographic products and perfectly normal spaces, Amer. Math. Monthly 78 (1971), 533-536.

[W] W. A. R. Weiss, Countably compact, perfectly normal spaces may or may not be compact, Notices Amer. Math. Soc. 22 (1975), A-334.

DEPARTMENT OF MATHEMATICS AND COMPUTER SCIENCE, UNIVERSITY OF SOUTH CAROLINA, COLUMBIA, SOUTH CAROLINA 29208 